\title{
Smooth Plurisubharmonic Functions Without Subextension
}

\author{
Eric Bedford ${ }^{1}$ and B.A. Taylor ${ }^{2}$ \\ ${ }^{1}$ Department of Mathematics, Indiana University, Bloomington, IN47405, USA \\ ${ }^{2}$ Department of Mathematics, University of Michigan, Ann Arbor, MI48109, USA
}

\section{§1. Introduction}

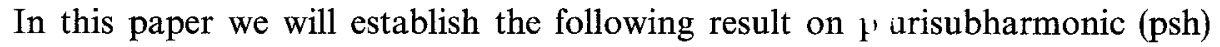
functions.

Theorem. Let $\Omega \Subset \mathbb{C}^{n}$ be a smoothly bounded domain. The, re exists .. smooth, psh function $\psi$ on $\Omega$ such that for any domain $\widetilde{\Omega}$ with $\operatorname{sen} \partial \Omega \neq \phi$, there is no function $\tilde{\psi}$ psh on $\widetilde{\Omega}$ such that $\tilde{\psi} \leqq \psi$ on $\widetilde{\Omega} \cap \Omega$.

It is evident, in particular, that $\psi$ cannot be extended to be psh in any larger domain, and thus we recover the known fact that $\Omega$ is a domain of existence for psh functions (see [1, 2]). However, since psh functions arise in complex analysis through their use in inequalities, the problem of subextension seems more appropriate than the problem of extension. Fornaess and Sibony [3] showed that there is a psh function on the ring domain $\left\{z \in \mathbb{C}^{n}: 1<|z|<R\right\}$ which cannot be subextended to the ball $\{|z|<R\}$. The function given in the Theorem improves this example by showing the function can be taken to be smooth and by showing that the failure of subextension is actually a local phenomenon.

The construction in the Theorem is based on Lemma 1 which shows that Lelong number can be both created and "propagated" by certain kinds of decrease. For example, if $\psi(z, w)$ is psh in a ball containing $(0,0)$ and if

$$
\psi(z, w) \leqq \log \left(\|w\|^{2}+e^{\left.-\frac{1}{|z|^{1 / 4}}\right)}\right.
$$

holds for $\operatorname{Re} z \geqq 0$, then $\psi(z, w)$ must have positive Lelong number on the variety $\{w=0\}$. The impossibility of subextension then arises from the Theorem of Siu which shows that the set where the Lelong number is $\geqq \varepsilon$ is a (global) variety.

Let us remark also that the Theorem gives a result on super-extension of $(1,1)$ currents. As was noted in [3], if $\psi$ is the function given in Theorem 1, then the (1,1)-form $S=d d^{c} \psi$ has the property:

if $\widetilde{\Omega}$ is any domain with $\widetilde{\Omega} \cap \partial \Omega \neq \phi$, then there is no positive, closed $(1,1)$-current $\tilde{S}$ on $\widetilde{\Omega}$ with $\tilde{S} \geqq S$ on $\Omega \cap \widetilde{\Omega}$. 
Acknowledgement. This work was carried out while the authors were visiting Universite de Provence at Marseille, and they wish to thank the Mathematics Department for its hospitality.

\section{§ 2. Propagation of Lelong Number}

We will let $z=z_{1} \in \mathbb{C}$ and $w=\left(z_{2}, \ldots, z_{n}\right)$ denote the coordinates of $(z, w) \in \mathbb{C}^{n}$. By $\|w\|$, we mean the Euclidean norm, $\|w\|^{2}=\left|z_{2}\right|^{2}+\ldots+\left|z_{n}\right|^{2}$. Our basic tool is the following.

Lemma 1. Let $\varphi(z, w)$ be $p s h, \varphi<0$, on a ball

$$
B=\left\{(z, w):|z|^{2}+\|w\|^{2}<1\right\} .
$$

Suppose there is an open cone $C_{\gamma}=\{(z, w):\|w\|<\gamma \operatorname{Re} z\}$ about the positive $\operatorname{Re}$ $z$-axis such that

$$
\varphi(z, w) \leqq \frac{1}{2} \log \left(\|w\|^{2}+r(z)^{2}\right), \quad(z, w) \in C_{\gamma},
$$
where $r(z)=r(|z|)$ is a monotone function such that $\frac{r(z)}{|z|^{m}} \rightarrow 0$ as $|z| \rightarrow 0$ for each
$m>0$. Then there exist constants $A, \eta>0$ such that

$$
\varphi(z, w) \leqq \eta \log \|w\|+A \quad \text { for all }|z|^{2}+\|w\|^{2}<\eta^{2} .
$$

Proof. We first show the hypotheses imply there is an estimate of the same form as (1),

$$
\varphi(z, w) \leqq \frac{\eta}{2} \log \left(\|w\|^{2}+\tilde{r}(z)^{2}\right)
$$

which holds for all $(z, w)$ in a smaller ball $|z|^{2}+\|w\|^{2}<\eta^{2}$. We consider two cases. First, suppose $\|w\|>|z|$. The point $(\|w\|, 0)$ lies in the cone $C_{\gamma}$. Consider the circle with center $(z, w)$ and boundary passing through $(\|w\|, 0)$,

Then

$$
(\zeta, \tau)=(z, w)+(\|w\|-z,-w) e^{i \theta}, \quad-\pi \leqq \theta \leqq \pi .
$$

and

$$
\|\tau\|=\|w\|\left|1-e^{i \theta}\right|=2\|w\|\left|\sin \frac{\theta}{2}\right| \leqq\|w\||\theta|
$$

$$
\begin{aligned}
\operatorname{Re} \zeta & =\operatorname{Re}\left(z\left(1-e^{i \theta}\right)\right)+\|w\| \cos \theta \\
& \geqq\|w\| \cos \theta-|z| \theta \geqq\|w\|(\cos \theta-\theta) .
\end{aligned}
$$

Hence, so long as

or

$$
\gamma(\cos \theta-|\theta|) \geqq|\theta|
$$

$$
\cos \theta \geqq|\theta|+\frac{1}{\gamma}|\theta|
$$

the points $(\zeta(\theta), \tau(\theta))$ lie in the cone $C_{\gamma}$. In particular, this happens on an interval $\left(-\theta_{0}, \theta_{0}\right)$, where $\theta_{0}$ depends only on $\gamma$. Thus, 


$$
\begin{aligned}
\varphi(z, w) & \leqq \frac{1}{2 \pi} \int_{-\pi}^{\pi} \varphi(\zeta(\theta), \tau(\theta)) d \theta \\
& \leqq \frac{1}{2 \pi} \int_{-\theta_{0}}^{\theta_{0}} \varphi(\zeta(\theta), \tau(\theta)) d \theta \\
& \leqq \frac{\theta_{0}}{2 \pi} \max _{|\theta| \leqq \theta_{0}} \log \left(\left(\tilde{r}(|\zeta(\theta)|)^{2}+\|\tau(\theta)\|^{2}\right) .\right.
\end{aligned}
$$

But, $|\zeta(\theta)| \leqq 2|z|+\|w\| \leqq 3\|w\|$, so $\tilde{r}(\zeta(\theta))^{2} \leqq\|w\|^{2}$ for sufficiently small $\|w\|$. Also, $\|\tau(\theta)\| \leqq 2\|w\|$, so we conclude $\varphi(z, w) \leqq \frac{\theta_{0}}{2 \pi} \log \left(5\|w\|^{2}\right)$, which implies (2) in this
case.

In the other case, $\|w\| \leqq|z|$, we can select $\lambda$ such that $\lambda \geqq 2+2 / \gamma$, and note that on the circle $\left(z+\lambda|z| e^{i \theta}, w\right)$, we have

$$
\gamma \operatorname{Re}\left(z+\lambda|z| e^{i \theta}\right) \geqq \gamma|z|(\lambda \cos \theta-1)=\gamma|z|[(\lambda-2) \cos \theta+(2 \cos \theta-1)] \geqq\|w\|
$$

whenever $\cos \theta \geqq 1 / 2$, or $|\theta| \leqq \pi / 3$. Thus, all the points on this circle lie inside $C_{\gamma}$ when $|\theta| \leqq \pi / 3$, so that

$$
\begin{aligned}
\varphi(z, w) & \leqq \frac{1}{2 \pi} \int_{-\pi}^{\pi} \varphi\left(z+\lambda|z| e^{i \theta}, w\right) d \theta \\
& \leqq \frac{1}{2 \pi} \int_{-\pi / 3}^{\pi / 3} \frac{1}{2} \log \left(\|w\|^{2}+r((1+\lambda)|z|)^{2}\right) d \theta \\
& =\frac{1}{6} \log \left(\|w\|^{2}+r((1+\lambda)|z|)^{2}\right)
\end{aligned}
$$

which is an estimate of the form (2). Consequently, (2) holds for $(z, w)$ near $(0,0)$.

Let $m\left(\rho_{1}, \rho_{2}\right)=\sup \left\{\varphi(z, w):|z|=\rho_{1},\|w\|=\rho_{2}\right\}, \quad$ and $\quad f(x, y)=\frac{1}{\eta} m\left(e^{x}, e^{y}\right)$. Then $f(x, y)$ is a convex function of $(x, y)$ defined for large negative $x$ and $y$, since $m$ is psh. The inequality (2) for $\varphi$ means that

$$
f(x, y) \leqq \frac{1}{2} \log \left(e^{2 h(x)}+e^{2 y}\right)=F(x, y)
$$

where $h(x)$ is a function such that $\frac{h(x)}{x} \rightarrow+\infty$ as $x \rightarrow-\infty$.

Suppose that an affine function $A x+B y+C$ satisfies

$$
A x+B y+C \leqq F(x, y)
$$

for all $x \leqq x_{0}, y \leqq y_{0}$. Then we claim $B \geqq 1$. To see this, note that

$$
A x+C \leqq \psi(x):=\inf _{y \leqq y_{0}} F(x, y)-B y
$$


We must have $B \geqq 0$, since if $B<0$, then $\psi(x)=-\infty$ for some finite $x$. If $B=0$, then $\psi(x)=h(x) \geqq A x+C$, contrary to the assumption that $\frac{h(x)}{x} \rightarrow+\infty$ as $x \rightarrow$ $-\infty$. The function $y \rightarrow F(x, y)-B y$ is convex. If $B>0$, then $y \rightarrow F(x, y)-B y$ tends to $+\infty$ as $y \rightarrow-\infty$. Thus the minimum of the function either occurs at $y=y_{0}$, or at the point $y<y_{0}$ where $\frac{\partial F}{\partial y}(x, y)=B$. A short calculation shows
that at this point, we must have

$$
e^{2 y}=\frac{B}{1-B} e^{2 h(x)} .
$$

Hence, if $0<B<1$ and $x$ is very large and negative, the minimum will occur at the point $y$ for which (5) holds, so the infimum in (4) is equal to

$$
h(x)-\frac{1}{2} \log (1-B) \text {. }
$$

But, this is impossible, for then

$$
A x+C \leqq h(x)+O(1) \text { as } \quad x \rightarrow-\infty
$$

contrary to our hypothesis that $\frac{h(x)}{x} \rightarrow+\infty$. Thus, we have $B \geqq 1$.

We can rewrite the affine function in (3) in the form $A\left(x-x_{0}\right)+B\left(y-y_{0}\right)+C$. Noting that when $B \geqq 1$, the minimum in (4) occurs for $y=y_{0}$, we have $A\left(x-x_{0}\right)+C \leqq F\left(x, y_{0}\right)$. Hence, the upper envelope of all affine functions satisfying (3) is at most $\left(y-y_{0}\right)+F\left(x, y_{0}\right)$. Since the convex function $f(x, y)$ is an upper envelope of affine functions satisfying (3), the last estimate of the lemma follows.

\section{§3. A Smooth Function without Subextension}

We first construct a continuous example and then modify it to give a smooth example.

For $0<\alpha<1 / 2$, let

$$
v(z)=v_{\alpha}(z)=\operatorname{Re}-\frac{1}{z^{\alpha}}=-r^{-\alpha} \cos \alpha \theta, \quad z=r e^{i \theta}, \quad-\pi \leqq \theta \leqq \pi, r>0
$$

and

$$
r(z)=e^{v(z)} .
$$

The function $v(z)$ is harmonic in $\mathbb{C} \backslash \mathbb{R}^{-}$, the complex plane with the negative real axis and 0 removed. It is continuous on $\mathbb{C} \backslash\{0\}$ and negative there, since $\alpha<1 / 2$. However, $v(z)$ is not subharmonic on the negative real axis. In fact, it is superharmonic there as locally it is the minimum of the two harmonic functions corresponding to different branches of $z^{\alpha}$. The function $r(z)$ is continuous, nonnegative, and vanishes to infinite order at $z=0$, since

$$
0 \leqq r(z) \leqq \exp \left(-\frac{\cos \alpha \pi}{|z|^{\alpha}}\right) .
$$


Next, let $\mathscr{U}$ be a neighborhood of 0 and $g: \mathscr{U} \rightarrow \mathbb{C}^{n-1}$ a function analytic for $z \in \mathscr{U}$ such that

$$
g(0)=0, \quad\left\|g^{\prime}(z)\right\| \leqq 1 / 4, \quad z \in \mathscr{U} .
$$

Consider the function $u$ defined by

$$
u(z, w)=u(z, w, \alpha, g)=\max \left\{v_{\alpha}(z), \log \|w-g(z)\|\right\} .
$$

The following proposition lists several properties of $u(z, w)$.

Proposition 2. The function $u$ of (6) satisfies

(i) $u$ is psh for $(z, w) \in\left[\left(\mathbb{C} \backslash \mathbb{R}^{-}\right) \cap \mathscr{U}\right] \times \mathbb{C}^{n-1}$ and on the open set

$$
\left\{(z, w) \in \mathscr{U} \times \mathbb{C}^{n-1}:\|w-g(z)\|>r(z)\right\} .
$$

(ii) $u(z, w) \leqq \frac{1}{2} \log \left(\|w-g(z)\|^{2}+r(z)^{2}\right)$.

(iii) $u$ is continuous on $\mathbb{C}^{n} \backslash(0,0)$, and $u(z, w) \rightarrow-\infty$ as $(z, w) \rightarrow(0,0)$.

Proof. For $z \in \mathscr{U}, z$ not on the negative real axis or $z=0, u(z, w)$ is clearly psh as the maximum of psh functions. The definition of $u(z, w)$ shows

$$
u(z, w)= \begin{cases}\log \|w-g(z)\| & \text { if }\|w-g(z)\|>r(z) \\ \log r(z) & \text { if }\|w-g(z)\| \leqq r(z)\end{cases}
$$

Thus, on the open set $\|w-g(z)\|>r(z), u$ is also psh because it is equal to $\log \|w-g(z)\|$, a psh function. The inequality of (ii) is also clear, since

$$
\begin{aligned}
u(z, w) & =\max \left\{\frac{1}{2} \log r(z)^{2}, \frac{1}{2} \log \|w-g(z)\|^{2}\right\} \\
& \leqq \frac{1}{2} \log \left(r(z)^{2}+\|w-g(z)\|^{2}\right) .
\end{aligned}
$$

The third assertion is clear.

The function $u(z, w)$ is essentially psh on the complement of the ball of radius 1 with center at $(-1,0)$. The only problem is that $u$ is only defined for $z$ near 0 . To get a globally defined function on a ring domain

$$
\Omega_{R}=\left\{(z, w): 1<|z+1|^{2}+\|w\|^{2}<(1+R)^{2}\right\},
$$

we want to take the function $u(z, w)$ for $(z, w)$ near 0 , and then modify it by some smooth function away from $(0,0)$. Precisely, we have the following.

Proposition 3. For any $\delta>0$, there exist constants $C_{1}, C_{2}>0$ such that the function $U(z, w)$ defined by

$$
\begin{array}{ll}
u(z, w) & \operatorname{Re} z \geqq-\delta \\
\max \left\{u(z, w), C_{1}-C_{2} \operatorname{Re} z\right\} & -\delta \geqq \operatorname{Re} z \geqq-2 \delta \\
C_{1}-C_{2} \operatorname{Re} z & -2 \delta \geqq \operatorname{Re} z
\end{array}
$$

is psh on $\Omega_{R}$. 
Now we note that the function $U(z, w)$ constructed on $\Omega_{R}$ is not smooth. However one may check that the singularities are locally of the form

$$
\begin{aligned}
U(z, w) & =\max \{S(z, w), h(z, w)\} \\
& =h(z, w)+\max \{0, S(z, w)-h(z, w)\}
\end{aligned}
$$

i.e., they are locally the maximum of a smooth psh function $S(z, w)$ and a pluriharmonic function $h(z, w)$. In other words, at a singular point

$$
U(z, w)=h(z, w)+\chi(S(z, w)-h(z, w))
$$

where $\chi(t)=\max (0, t)$. If we replace $\chi$ by a $C^{\infty}$ convex and increasing function $\tilde{\chi}$ with $\tilde{\chi}(t)=0$ for $t \leqq-\varepsilon$ and $\tilde{\chi}(t)=t$ for $t \geqq \varepsilon$, then the resulting function $\tilde{U}$ will be $C^{\infty}$ and psh, and $|\tilde{U}-U| \leqq 2 \varepsilon$. Further, the function is changed only on the set $\{-\varepsilon<S-h<\varepsilon\}$ so that if $\varepsilon$ is taken to be small, $\tilde{U}=U$ holds near any other singularity.

We will now select a particular curve $g(z)$ so that the function $\tilde{U}$ constructed above does not subextend. Choose real numbers $\left(\beta_{2}, \ldots, \beta_{n}\right)$ such that the curve

$$
c(t)=\left(e^{i t}, e^{2 \pi i \beta_{2} t}, \ldots, e^{2 \pi i \beta_{n} t}\right), \quad-\infty<t<+\infty,
$$

is dense in the $n$-torus. Then, for $\varepsilon$ a small positive number, let $g(z)$ be the analytic curve defined for $|z|<1$ by $g(z)=\left(g_{2}(z), \ldots, g_{n}(z)\right)$, where

$$
g_{i}(z)=\varepsilon(1+z)^{\beta_{i}}-\varepsilon(1+z)
$$

(and the principle branch of $(1+z)^{\beta_{i}}$ is used). The conditions $g(0)=0,\left\|g^{\prime}(z)\right\| \leqq \frac{1}{4}$ are satisfied on $\{\operatorname{Re} z \geqq-2 \delta,|z|<R\}$ if $\delta>0$ is sufficiently small. Thus, we obtain the function $\tilde{U}$ as above.

Proposition 3. The function $\tilde{U}$ constructed as above is smooth and psh on the domain $\Omega_{R}$, and for every $\gamma>0$, there is no psh function $\phi$ on $\left\{|z|^{2}+\|w\|^{2}<(1+\gamma)^{2}\right\}$ such that $\phi(z, w) \leqq \tilde{U}(z, w)$ holds on the set

$$
\widetilde{C}_{\gamma}=\left\{(z, w): \operatorname{Re} z>0,\|w\|<\gamma,|z|^{2}+\|w\|^{2}<(1+\gamma)^{2}\right\} .
$$

Proof. We have already seen that $\tilde{U}$ is smooth and psh on $\Omega_{R}$. If $\phi$ exists, then we have the estimate

$$
\phi(z, w) \leqq \tilde{U}(z, w) \leqq \frac{1}{2} \log \left[\|w-g(z)\|^{2}+r(z)^{2}\right]
$$

on $\widetilde{C}_{\gamma}$. If we make the local biholomorphic change of coordinates $z^{\prime}=z, w^{\prime}=w$ $-g(z)$, we have

$$
\phi\left(z^{\prime}, w^{\prime}\right) \leqq \frac{1}{2} \log \left[\left|w^{\prime}\right|^{2}+r(z)^{2}\right]
$$

for all the points $\left(z^{\prime}, w^{\prime}\right)$ in a cone $C_{\gamma}$ about the $\operatorname{Re} z^{\prime}$ axis in some small ball about $(0,0)$. Then, by Lemma 1 , there exists $\eta>0$ such that

$$
\varphi(z, w) \leqq \eta \log \|w-g(z)\|+\mathcal{O}(1)
$$

for all $(z, w)$ in a neighbourhood of $(0,0)$. Thus, the Lelong number of $\varphi$ is at least $\eta$ at every point of the variety $w=g(z)$ near to $(0,0)$. 
By Siu's theorem [4], the set of points in $\left\{|z|^{2}+\|w\|^{2}<(1+\gamma)^{2}\right\}$ where the Lelong number is $\geqq \eta$ is an analytic variety, $V$. Since it contains the part of the curve $w=g(z)$ near $(0,0)$, it also contains all the points $(z, w)$ one can connect to $(0,0)$ by analytic continuation along curves in the set

$$
S=\{w=g(z)\} \cap\left\{|z|^{2}+\|w\|^{2}<(1+\gamma)^{2}\right\} .
$$

However, we claim that this set is contained in no (proper) analytic variety. For, we can clearly follow a path in $S$ to a small neighborhood of the branch point $z=-1, w=0$. Then for small $\rho$, analytic continuation on the path with $z(t)=-1+\rho e^{i t},-\infty<t<+\infty$, shows that $V$ contains all the points $(z(t), w(t))$ where

$$
w(t)=-\varepsilon \rho e^{i t}(1, \ldots, 1)+\varepsilon\left[\rho^{\beta_{2}} e^{2 \pi i \beta_{2} t}, \ldots, \rho^{\beta_{n}} e^{2 \pi i \beta_{n} t}\right] .
$$

This set is not contained in any (proper) variety in a neighborhood of $(-1,0)$, since under the biholomorphic map $z^{\prime}=(1+z), w^{\prime}=w+\varepsilon(1+z)=w+\varepsilon z^{\prime}$ its image is a dense subset of the distinguished boundary of the polydisk with center at $(0,0)$ and polyradius $\left(\rho, \varepsilon \rho^{\beta_{2}}, \ldots, \varepsilon \rho^{\beta_{n}}\right)$. This is a contradiction so no such function $\phi$ can exist.

We remark that in the proof one could use instead of Siu's theorem a weaker version due to Skoda [5], which asserts that this set is contained in a variety.

Proof of the Theorem. We use the notation

$$
A\left(\left(z_{0}, w_{0}\right), r_{1}, r_{2}\right)=\left\{(z, w) \in \mathbb{C}^{2}: r_{1}^{2}<\left|z-z_{0}\right|^{2}+\left\|w-w_{0}\right\|^{2}<r_{2}^{2}\right\} .
$$

If $\Omega$ is smoothly bounded, then there is a sequence of domains

$$
A_{j}=A\left(\left(z_{0}^{j}, w_{0}^{j}\right), r_{1}^{j}, r_{2}^{j}\right)
$$

such that $\Omega \subset \cap A_{j}$ and there is a point $p_{j} \in \partial \Omega \cap \partial A_{j}$. Clearly we can choose the $A_{j}$ such that the set $\left\{p_{j}\right\}$ is dense in $\partial \Omega$. By Proposition 3 there exists $\psi_{j}$ which is psh and smooth and cannot be locally subextended over $p_{j}$. Without loss of generality, we may assume $\psi_{j}<0$. If we choose $\varepsilon_{j}>0$ such that $\psi=\sum \varepsilon_{j} \psi_{j}$ converges in $C^{\infty}(\Omega)$, then $\psi$ cannot be subextended over any neighborhood of $\partial \Omega$. For if $\widetilde{\Omega}$ and $\tilde{\psi}$ are given, then there exists $p_{j} \in \widetilde{\Omega} \cap \partial \Omega$, but $\tilde{\psi} \leqq \varepsilon_{j} \psi$ holds on $\widetilde{\Omega} \cap \partial \Omega$, which is a contradiction.

\section{References}

1. Bedford, E., Burns, D.: Domains of existence of plurisubharmonic functions. Math. Ann. 238, 67-69 (1978)

2. Cegrell, U.: Removable singularities for plurisubharmonic functions and related problems. Proc. Lond. Math. Soc., III. Ser. 36, 310-336 (1978)

3. Fornaess, J.E., Sibony, N.: Plurisubharmonic functions on ring domains. Preprint

4. Siu, Y.T.: Analyticity of sets associated to Lelong numbers and the extension of closed positive currents. Invent. Math. 27, 53-156 (1974)

5. Skoda, H.: Sous-ensembles analytiques d'ordre fini ou infini dans $\mathbb{C}^{n}$. Bull. Soc. Math. France 100, 353-408 (1972) 